\title{
Synthesis of Self-Assembled Redox-Active-Polymer on Electrode Surface for Biosensor Application
}

\author{
Shu Taira, Eiichi Tamiya ${ }^{1}$, Kenji Yokoyama ${ }^{2, *}$ and Eiichiro Ichiishi** \\ School of Materials Science, Japan Advanced Institute of Science and Technology, \\ 1-1 Asahidai, Nomi, Ishikawa 923-1292, Japan \\ ${ }^{1}$ Department of Applied Physics, Graduate School of Engineering, Osaka University, \\ 2-1 Yamadaoka, Suita, Osaka 565-0871, Japan \\ ${ }^{2}$ Research Center of Advanced Bionics, National Institute of Advanced Industrial Science and \\ Technology (AIST), AIST Tsukuba Central 4, 1-1-1 Higashi, Tsukuba 305-8562, Japan
}

(Received July 24, 2008; accepted September 5, 2008)

Key words: redox active polymer, self-assembled monolayer, surface polymerization

To develop a stable electrochemical biosensor device, a gold electrode surface was modified with a polymerization initiator by the self-assembled monolayers (SAMs) method for surface polymerization. Poly(vinylferrocen-co-acrylamide) as a redox active polymer was living-radical-polymerized at the electrode surface, avoiding a homogeneous polymerization in a solution and physical adsorption of free polymer on the electrode. The living-radical-polymerized redox active polymer showed stable electrochemical response and catalytic enzyme response. The SAM-based synthesis and immobilization of the redox active polymer was carried out. The redox-active-polymermodified electrode showed a stable electrochemical response after several potential sweeps. The current value increased with increasing in polymerization time. The surface polymerization can be used to control the polymer chain growth on the electrode surface.

\section{Introduction}

Synthetic and intravital polymers are usable material in the analytical ${ }^{(1-3)}$ and medical fields ${ }^{(4,5)}$ owing to the multiple molecular interactions compared with smaller compounds. Particularly, reduction/oxidation (redox) active polymers have been studied as electron transfer mediators for medical biosensors. ${ }^{(6-10)}$ These polymers can be used to construct the reusable sensor. The immobilization of an electron transfer mediator and enzyme is very important to avoid desorption from the electrode surface. Immobilization based on the physical adsorption or adhesion of a redox active polymer and an enzyme on an electrode has been carried out. ${ }^{(8,9)}$ This method suffers from desorption or elimination of the polymer and enzyme from the electrode during measurement.

*Corresponding author: e-mail: ke-yokoyama@aist.go.jp,

${ }^{* *}$ Corresponding author: e-mail: ichiishi@jaist.ac.jp 
Self-assembled monolayers (SAMs) have been studied for the chemical immobilization of various materials on the surface. SAMs of organic molecules on $\mathrm{Au}(111)$ are becoming increasingly important not only in the areas of fundamental surface science and interfacial electrochemistry ${ }^{(11,12)}$ but also in other areas. For example, in many biomedical applications, SAMs separate a metal surface from a solution. ${ }^{(13-16)}$ Alkanethiols adsorbed on gold are closely packed via thiolate-gold covalent bonds and van der Waals forces between the alkyl chains. This can prevent the desorption of materials from the substrate surface. ${ }^{(17,18)}$ Our previous work showed that a self-assembled polymer is easily immobilized on a gold substrate and can be applied to a biosensor. ${ }^{(19-21)}$ SAM-based and ion-complex enzyme electrodes have showed that a catalytic current value increases upon glucose addition. Also, the electrode showed a stable response after several scans. Therefore, the desorption of the materials from the electrode was prevented when the SAM and ion complex were used for immobilization. The SAM-based and ion-complex immobilization of redox active polymer and enzyme could lead to the construction of reusable enzyme sensors. ${ }^{(22)}$ However, we were constrained to handle several steps before the immobilization of the polymer on the surface. Synthesis of polymer films from only the substrate surface via SAM-based surface living radical polymerization can simplify the experimental procedure and can avoid unworthy polymerization in solution. Scheme 1 shows that SAM-based surface living radical polymerization produces only a polymer chain covalently immobilized to the electrode surface, whereas, conventional solution polymerization yields both immobilized and free polymers. The surface confined redoxactive-polymer can smoothly achieved electron transfer. In this study, we report that a gold electrode was modified with a redox active polymer using the SAMs technique and surface living radical polymerization. 4-(Chloromethyl)benzoic acid as a polymerization initiator was attached to cystamine which was immobilized on the gold electrode surface using the SAMs technique. Benzyl chloride groups of 4-(chloromethyl)benzoic acid bonded to the surface form a controlled number of radicals by atom transfer to a $\mathrm{Cu}-$ 2,2'-bipyridyl as catalyst. ${ }^{(23)}$ Equilibrium is maintained between the active and inactive chain ends, $\mathrm{R}^{\bullet}$ and $\mathrm{RCl}$ with the equilibrium favoring the inactive species, minimizing termination and radical transfer reactions, therefore the length of the polymer chain can be controlled by adjusting the polymerization time. Hence, polymerization only occurs on the electrode surface. The chemical immobilized redox-active-polymer by SAM has flexibility unlike the physical immobilization of the polymer via the crosslinking reagent. ${ }^{(9)}$ The obtained electrode shows a stable electrochemical response.

\section{Experimental}

\subsection{Materials}

Cysteamine, 4-(chloromethyl)benzoic acid, and vinylferrocene (Vfc) were purchased from Aldrich (USA). Copper(I), ${ }_{\mathrm{D}}(+)$-glucose 2,2'-bipyridyl chloride and dimethylformamide, acrylamide (AAm) monomer, sodium dihyrogenphosphate dihydrate, $N$-methylmorpholine (NMM), dimethylformamide (DMF), and disodium hydrogenphoshate 12-water were purchased from Wako Pure Chemical Industries, Ltd. (Osaka, Japan). The glucose solution was equilibrated overnight prior to measurements. 


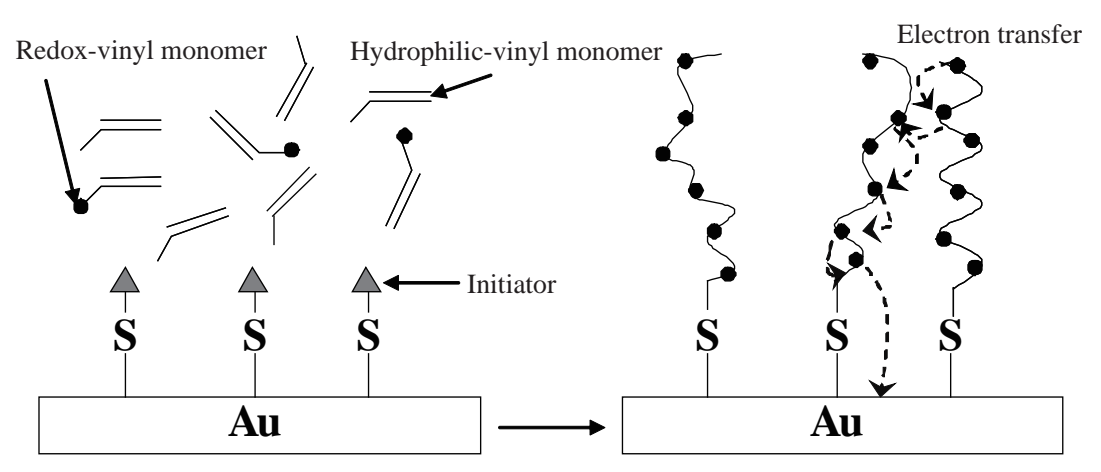

(a)
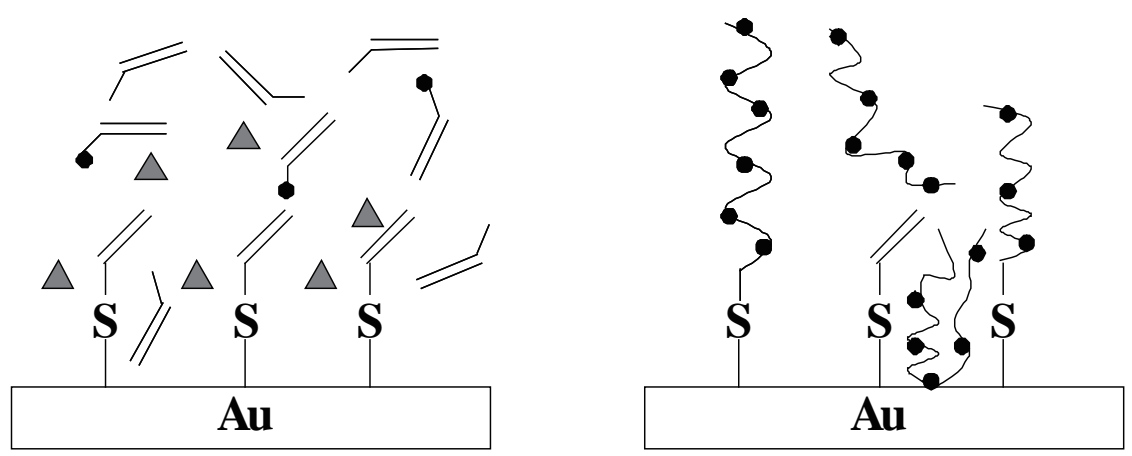

(b)

Scheme 1. Schematic illustration of (a) SAM-based surface-confined living radical polymerization and (b) solution polymerization.

Glucose oxidase (GOx) from Aspergillius niger type XS 25,000 units/g was purchased from Sigma (USA). GO $x$ content was determined to be $6.71 \times 10^{-9}$ [mol/mg-solid] by measuring the FAD content. The FAD concentration was determined from the absorbance at $450 \mathrm{~nm}$ using $\varepsilon 450=14.1 \mathrm{mM}^{-1} \mathrm{~cm}^{-1}$.(24) The ratio of GOx bound to total FAD molecules was measured using gel filtration chromatography (TOSOH HPLC, TSK-gel G3000SWXL, Japan) and was found to be 86\%. Benzotriazole-1-yl-oxy-trispyrrolidino-phosphonium hexafluorophosphate (PyBOP) and $N$-hydroxybenzotriazole (HOBt) were purchased from Merck (Tokyo, Japan). Ultrapure water (18.3 M $\Omega-\mathrm{cm})$ from Millipore was used in all the experiments.

\subsection{Homogeneous polymerization of redox active polymer}

4-(Chloromethyl)benzoic acid (3.0 mM) as initiator, Vfc (17 mM), AAm (0.86 M), copper(I) chloride $\left(2.9 \times 10^{-1} \mathrm{mM}\right)$ and $2,2^{\prime}$-bipyridyl $\left(7.9 \times 10^{-2} \mathrm{mM}\right)$ as catalysts 
were dissolved in DMF. Radical polymerization was carried out at $130^{\circ} \mathrm{C}$ for 3 or $6 \mathrm{~h}$. The molecular weight of the obtained redox active polymer was determined by Gel Permeation Chromatography (GPC). GPC was carried out using Tosoh TSKgel G4000PWXL column and a UV detector $(254 \mathrm{~nm})$ at $40^{\circ} \mathrm{C}$. Poly(ethylene oxide) (PEO) (MW: 920,000, 540,000, 250,000, 140,000, 100,000, 50,000, 240,000, 2,000 and 200) was used to prepare calibration curve.

\subsection{Surface-initiated radical copolymerization of $V f_{C}$ and AAm}

A gold disk electrode of $6 \mathrm{~mm}$ diameter was used. The gold electrode was rinsed with methanol, followed by soaking in cysteamine solution $(1 \mu \mathrm{M})$ for $2 \mathrm{~h}$. The amino group of cysteamine on the gold electrode surface was modified with 4-(chloromethyl)benzoic acid (4.6 mM) using PyBOP (9.2 mM), HOBt (9.2 mM), and NMM (9.2 mM) (Fig. 1(a)). Then, Vfc (18 mM), AAm (0.9 M), copper(I) chloride (0.3 mM), and 2,2' -bipyridyl $\left(7.9 \times 10^{-2} \mathrm{mM}\right)$ were sonicated for $1 \mathrm{~min}$ to accelerate the dissolution in DMF. 4-(Chloromethyl)benzoic acid-immobilized electrode was immersed in this solution. Surface radical polymerization was allowed to proceed at $130^{\circ} \mathrm{C}$ for 3,6 and $12 \mathrm{~h}$. After the reaction, the electrode was washed several times with distilled water to remove the physically adsorbed monomer reagent. A schematic description of the polymerization of the redox active polymer on the gold electrode surface is given in Fig. 1(b).

\subsection{Electrochemical measurement}

A three-electrode configuration was used. A SAM-based redox-active-polymermodified electrode, a platinum wire, and $\mathrm{Ag} / \mathrm{AgCl} / \mathrm{KCl}$ were used as the working, auxiliary, and reference electrodes, respectively. All the potentials herein are quoted with respect to these electrodes. Unless otherwise stated, cyclic voltammetry (CV) was carried out in a deoxygenated phosphate buffer $(0.1 \mathrm{M} \mathrm{pH} 7.0)$ with a computercontrolled potentiostat (CV-50W BAS) at room temperature.

\section{Results and Discussion}

\subsection{Characterization of homogeneous polymerized redox polymer}

We investigated the effect of polymerization time on the molecular weight $(\mathrm{Mw})$ of the redox active polymers. GPC elution curves showed that the Mw of the redox active polymers did not increase with the increasing in the polymerization time. The Mw of both redox active polymers was estimated as Mw 450,000 (Fig. 2). The result indicated that the reaction rate was very fast and the termination reaction occurred in a homogeneous system.

Homogeneous polymerized redox active polymers were easily dissolved in buffer solution on showed a typical electrochemical response: polymerization time of 3 on 6 $h$, respectively. We investigated the effect of polymerization time on the peak-to-peak potential separation $(\Delta E \mathrm{p})$ of redox active polymer. Both $\Delta E \mathrm{p}$ values showed almost same $(\sim 80 \mathrm{mV})$ which did not correlate with polymerization time.

The catalytic electrochemistry of GOx with the redox-active-polymer-based enzyme electrode with glucose was investigated. A catalytic current was observed upon glucose 

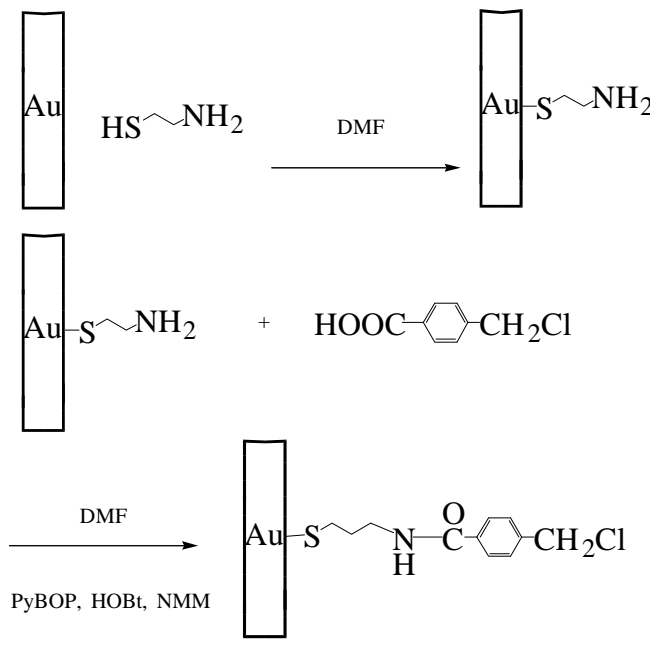

(a)
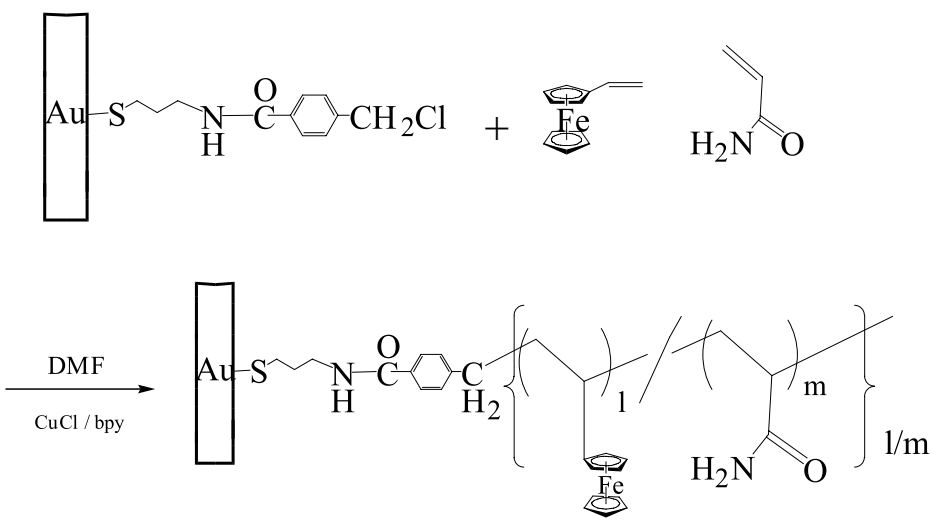

(b)

Fig. 1. (a) SAM-based immobilization of cysteamine and 4-chloromethyl benzoic acid on gold surface, (b) SAM-based co-polymerization on gold surface.

addition (Fig. 3). The peak current value of the catalytic response showed a positive shift compared with that without the glucose substrate. The phenomenon occurred owing to the increase in concentration of the reduced mediator at the electrode of as high as the substrate concentration $(0.1 \mathrm{M})$. Catalytic current values at $+280 \mathrm{mV}$ increased to 2 (Fig. 3(a)) and 1.4 (Fig. 3(b)) times, respectively. The copolymer of Vfc and AAm has redox and mediator abilities. 


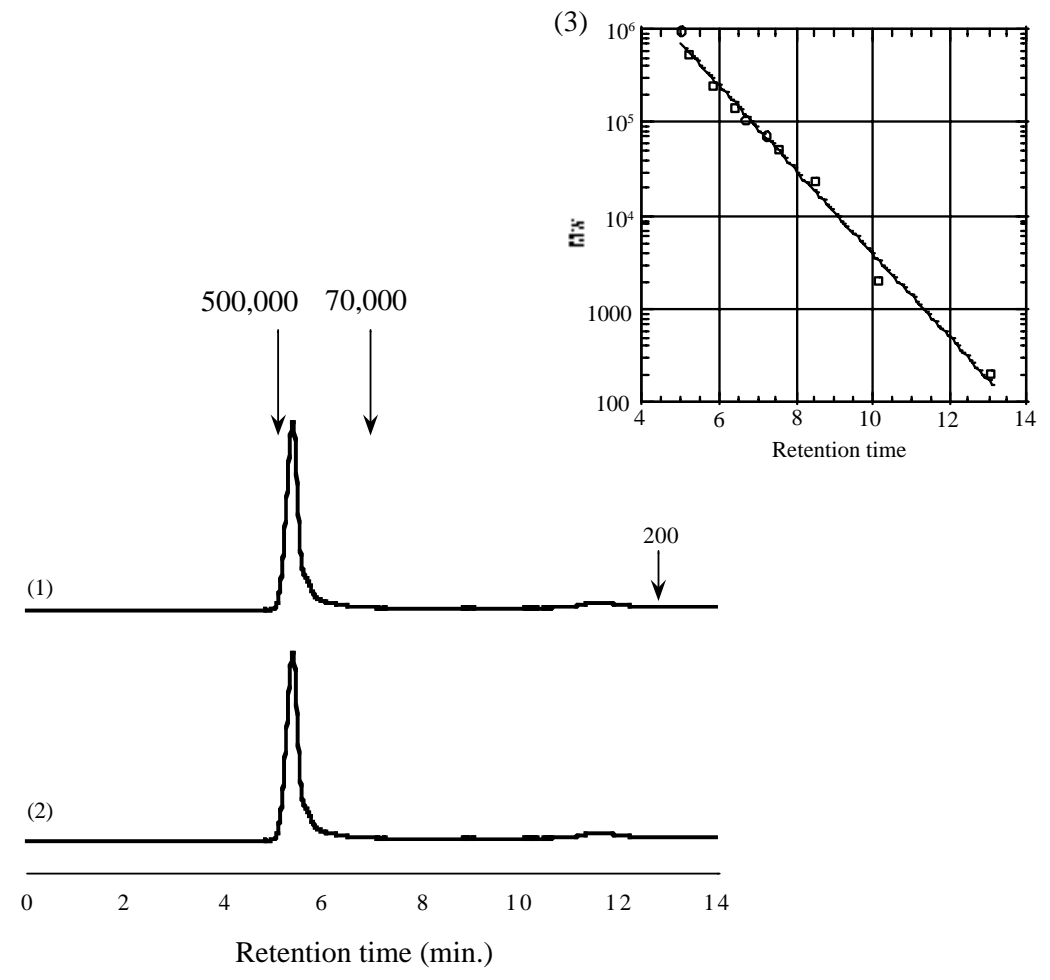

Fig. 2. GPC elution curves of poly(VFc-co-AAm); polymerization time of (1) 3 on (2) 6 h. (3) GPC calibration curve of standard PEO.

\subsection{Stability of electrochemical reaction for SAM-based redox-active-polymer- immobilized electrode}

The redox-active-polymer-modified electrode was characterized by CV. Figure 4 shows typical cyclic voltammograms of the redox active polymer modified electrode that was surface-polymerized for 3 (Fig. 4(a)) on 6 h (Fig. 4(b)). To remove the physically adsorbed Vfc monomer, the electrode potential was held at $500 \mathrm{mV}$ for $8 \mathrm{~min}$, followed by changing the buffer solution; viz., Vfc monomer become hydrophilic by maintaining the positive potential on the electrode. Thus the only chemically adsorbed redox active polymer using SAM was retained on the electrode. Both electrodes showed a stable response after the 5th measurement because of the no difference in the response between the 5th and the 6th measurements (Figs. 4(a) and 4(b) (lines (3) and (4)), although the response decreased compared with the 1st response. We deduce that the first large abatement occurred owing to the absolute elimination of the excess physical adsorption of redox monomers on the surface. For Fig. 4(b), the rate of change of the electrochemical response was smaller than that shown in Fig. 4(a). We compared the 


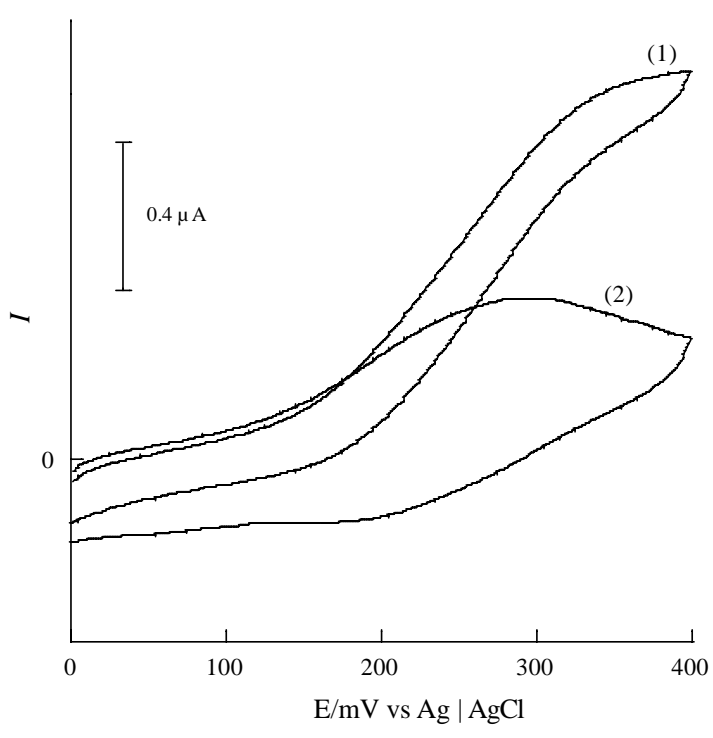

(a)

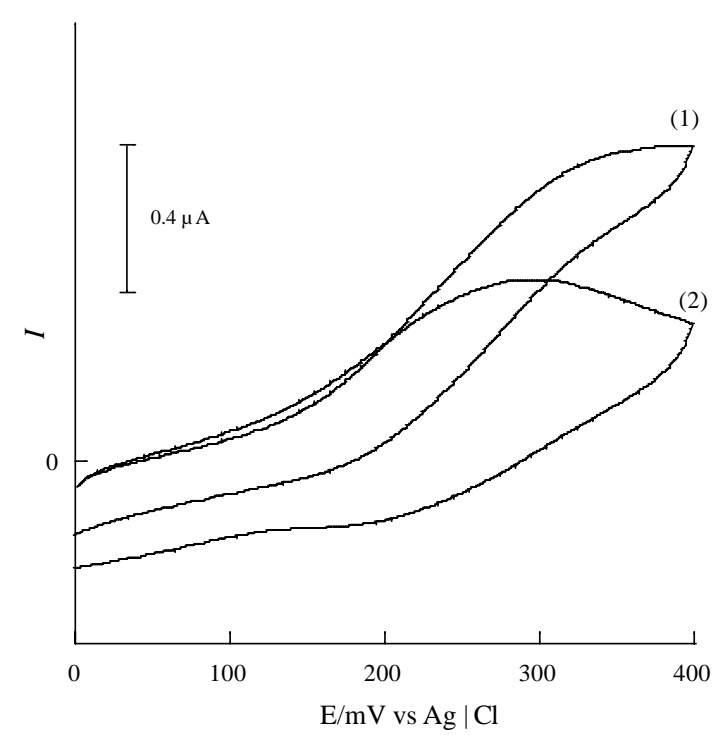

(b)

Fig. 3. Catalytic electrochemistry of enzyme with $2 \mathrm{mg}$ of redox active polymer: polymerization time of (a) 3 on (b) $6 \mathrm{~h}$. Cyclic voltammetries were carried out at scan rate of $5 \mathrm{mV} / \mathrm{s}$ in $0.1 \mathrm{M}$ phosphate buffer (pH 7.0) and 0.1 M glucose (1) with and (2) without $33.5 \mu \mathrm{M} \mathrm{GOx.}$

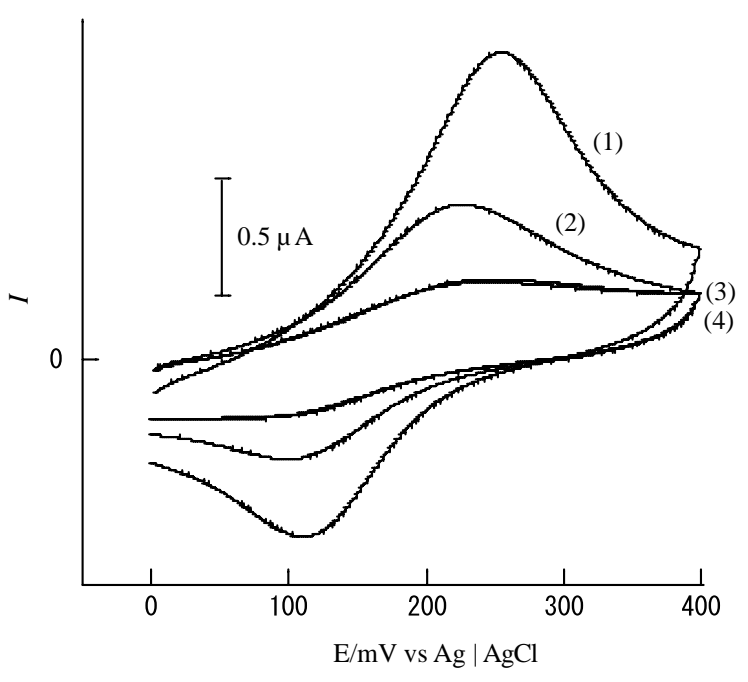

(a)

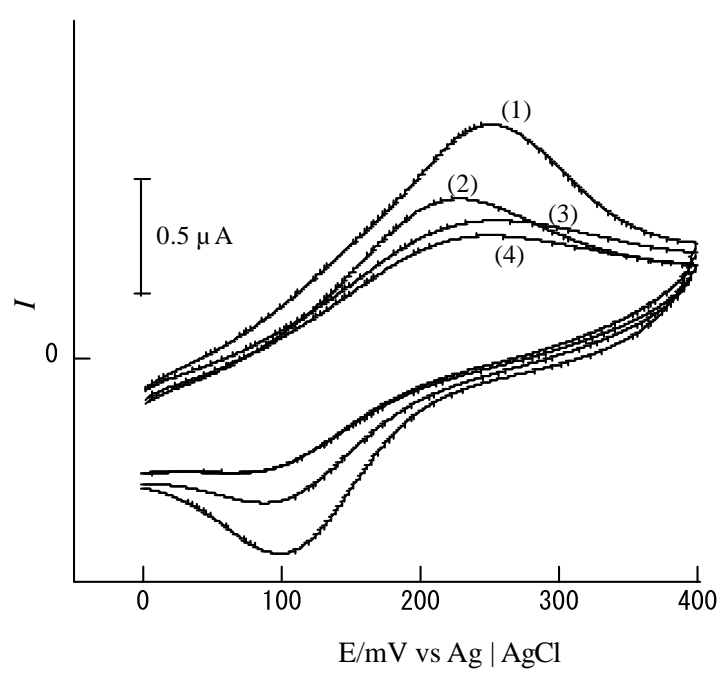

(b)

Fig. 4. CV curves of SAM of poly(VFc-co-AAm)-immobilized Au electrode: polymerization time of (a) 3 on (b) $6 \mathrm{~h}$. Cyclic voltammetries were carried out at a scan rate of $5 \mathrm{mV} / \mathrm{s}$ in phosphate buffer (pH 7.0). The electrode was held at $500 \mathrm{mV}$ for $480 \mathrm{~s}$ after the 1 st measurement. The buffer solution was changed after the 1st, 3rd, and 5th measurements. CV measurements of (1) 1st, (2) 2nd, (3) 5th, and (4) 6th. 
1st oxidation peak value and that of the 6th measurement. The abatement ratios were 74\% (Fig. 4(a)) and 47\% (Fig. 4(b)), respectively. This presumably indicated that the longer polymerization can avoid the excess physical adsorption of the monomer reagent namely the polymer density increases with increasing polymerization time. Nonetheless, this decrease in response owing to settling redox ability of Vfc does not affect the use of this electrode as sensor device. The oxidation and reduction peaks of Figs. 4(a) and 4(b) at 6th measurement were observed at $(+243,+102 \mathrm{mV})$ and $(+246,+100 \mathrm{mV})(\mathrm{vs}$ $\mathrm{Ag} / \mathrm{AgCl})$, respectively. For $\Delta E \mathrm{p}$ of the redox-active-polymer-immobilized electrode, these values (141 and $146 \mathrm{mV}$ ) increased in comparison with that of the homogeneous system $(\sim 80 \mathrm{mV})$ at the given scan rate. Electron transfer on the redox-active-polymerimmobilized electrode was slower than that on the homogeneous one owing to the limitation of polymer flexibility by the SAM-based immobilization.

\subsection{Effect of polymerization time}

The effect of surface polymerization time was investigated by CV. In the homogeneous polymerization system, the obtained polymers showed almost the same $\mathrm{Mw}$ and $\mathrm{CV}$ responses. These results are suggested that the termination reaction occurred in the early stage of polymerization. We carried out surface living polymerization for 3 and $6 \mathrm{~h}$ to confirm the effect of polymerization time on the electrochemical response. We compared these stable electrochemical responses after the 6th measurement (Fig. 5). These CV curves showed the current value dependence on the polymerization time. Polymer chain growth on an electrode depended on the polymerization time. For this reason, surface-initiated polymerization occurred more slowly than the homogeneous system. In addition, a very long polymerization time (12 h) did not increase the electrochemical response compared with the polymerization time of $6 \mathrm{~h}$,

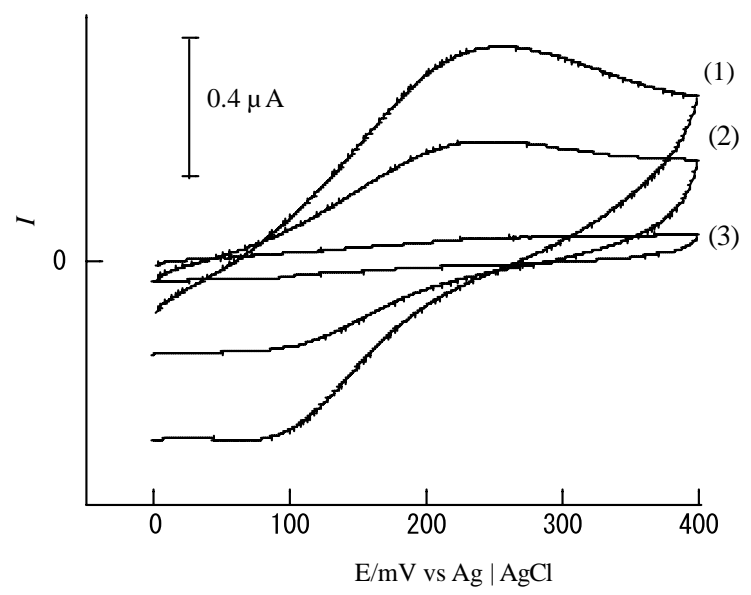

Fig. 5. CV curves of SAM of poly(VFc-co-AAm) immobilized Au electrode: surface polymerization time of (1) 6, (2) 3 on (3) 0 h. Cyclic voltammetries were carried out at a scan rate of $5 \mathrm{mV} / \mathrm{s}$ in $0.1 \mathrm{M}$ phosphate buffer (pH 7.0). 
because the termination reaction also occurred for excess long synthesis time (data not shown). During this time, the electrochemical current value increased with increasing polymerization time within $6 \mathrm{~h}$. It is noteworthy that the amount of redox active polymer on an electrode surface can be controlled by adjusting the polymerization time owing to the adoption of the surface living polymerization method.

\section{Conclusions}

In this work, our electrode can retain redox-active-polymer after washing owing to the covalently attachment between the initiator and electrode surface by SAM. These polymer-immobilized electrodes showed a stable response after six measurements. We could control the electrochemical response by adjusting the surface polymerization time. Particularly, the electrode that is polymerized for $6 \mathrm{~h}$ showed a stable electrochemical response (Fig. 5, line (1)). The limit for the mediator active has these SAM-based redoxactive-polymer-immobilized electrodes. In the homogeneous system, we demonstrated the redox-active-polymer-mediated enzyme reaction; we expect that future optimization will enable us to analyze the enzyme reaction. Our current effort to solve this problem is to elucidate the factors involved in the effect of polymer surface density and the ratio of Vfc to AAm in one polymer chain. In this time, we synthesized poly(VFc-co-AAm) (i.e., the molar ratio of Vfc to AAm was 1:50). Thus, the amount of redox active sites in the polymer chain was presumably small. Nevertheless, we foresee a large potential of living surface polymerization for the very practical preparation of reusable redox active electrode, because this electrode shows stable electrochemical response after several measurements. Hence, it is expected that this electrode can detect the reaction between the enzyme and substrate by detecting the electron transfer between the enzyme and redox polymer on the electrode surface. ${ }^{(9,22)}$

\section{Acknowledgment}

We thank the members of the Research Center of Advanced Bionics and the Ichiishi laboratory in JAIST, Dr. T. Endo of the Tokyo Institute of Technology, Dr. S. Koide of AIST, and Dr. Y. Morita of Kinki University for the helpful discussions.

\section{References}

1 M. J. Kastantin, S. Li, A. P. Gadre, L.-Q. Wu, W. E. Bentley, G. F. Payne, G. W. Rubloff and R. Ghodssi: Sens. Mater. 15 (2003) 295.

2 S. Taira, Y. Z. Du, Y. Hiratsuka, T. Q. P. Uyeda, N. Yumoto and M. Kodaka: Biotechnol. Bioeng. 99 (2008) 734.

3 Y. Miura: J. Polym. Sci., Part A: Polym. Chem. 45 (2007) 5031.

4 T. Ooya, M. Eguchi and N.Yui: J. Am. Chem. Soc. 125 (2003) 13016.

5 S. Arai, Y. Morinaga, T. Yoshikawa, E. Ichiishi, Y. Kiso, M. Yamazaki, M. Morotani, M. Shimizu, T. Kuwata and S. Kaminogawa: Biosci. Biotechnol. Biochem. 66 (2002) 2017.

6 H. Muguruma, Y. Kase and H. Uehara: Anal. Chem. 77 (2005) 6557. 
7 J. Niedziolka, E. Rozniecka, J. Stafiej, J. Sirieix-Plenet, L. Gaillon, D. di Caprio and M. Opallo: Chem. Commun. 23 (2005) 2954.

8 H. B. Yildiz, J. Castillo, D. A. Guschin, L. Toppare and W. Schuhmann: Microchim. Acta 159 (2007) 27.

9 S. Koide and K. Yokoyama: J. Electroanal. Chem. 468 (1999) 193.

10 Y. Liu, A. G. Erdman and T. Cui: Sens. Actuators, A 136 (2007) 540.

11 J. Lipkowski and P. N. Ross (eds.): Adsorption of Molecules at Metal Surfaces (VCH Publishers, New York, 1992).

12 H. O. Finklea, A. J. Bard and I. Rubinstein (eds.): Electroanalytical Chemistry (Marcel Dekker, New York, 1996).

13 B. Fang, X.-H. Deng, X.-W. Kan, H.-S. Tao, W.-Z. Zhang and M.-G. Li: Anal. Lett. 39 (2006) 697.

14 T. A. Lewington, M. R. Alexander, G. E. Thompson and E. McAlpine: Surf. Eng. 18 (2002) 228.

15 W. Song, M. Okamura, T. Kondo and K. Uosaki: J. Electroanal. Chem. 554 (2003) 385.

16 H. Kimura-Suda, D. Y. Petrovykh, M. J. Tarlov and L. J. Whitman: J. Am. Chem. Soc. 125 (2003) 9014.

17 S. Taira and K.Yokoyama: Biotechnol. Bioeng. 88 (2004) 35.

18 E. M. Boon, J. E. Salas and J. K. Barton: Nature Biotechnol. 20 (2002) 282.

19 S. Taira and K. Yokoyama: Anal. Sci. 19 (2003) 177.

20 S. Taira and K. Yokoyama: Anal. Sci. 20 (2004) 1.

21 S. Taira and K.Yokoyama: Biotechnol. Bioeng. 89 (2005) 835.

22 S. Taira, E.Tamiya and K. Yokoyama: Electrochem. 69 (2001) 940.

23 X. Huang, L. J. Doneski and M. J. Wirth: Anal. Chem. 70 (1998) 4023.

24 H. J. Bright and Q. H. Gibson: J. Biol. Chem. 242 (1967) 994. 